\title{
Anthropometric, body composition, and somatotype characteristics of Japanese young women: Implications for normal-weight obesity syndrome and sarcopenia diagnosis criteria
}

\author{
TOMOHIRO YASUDA ${ }^{1,2, *}$ \\ ${ }^{1}$ School of Nursing, Seirei Christopher University, Shizuoka, Japan \\ ${ }^{2}$ Graduate School of Medicine, Dokkyo Medical University, Tochigi, Japan \\ *Corresponding address: Tomohiro Yasuda, PhD; School of Nursing, Seirei Christopher University, 3453, Mikatahara, Kita-Ku, Hamamatsu, \\ Shizuoka 433-8558, Japan; Phone: +81 53439 1417; Fax: +81 53439 1406; E-mail: tomohiro-y@seirei.ac.jp
}

(Received: December 26, 2018; Revised manuscript received: March 24, 2019; Accepted: April 23, 2019)

\begin{abstract}
Aims: The purpose of this study was to examine the anthropometric, body composition, and somatotype characteristics of Japanese young women and to focus on normal-weight obesity syndrome and sarcopenia diagnosis criteria. Methods: A total of 124 Japanese university freshmen women were measured at body mass index (BMI), percent body fat and skeletal muscle index (SMI), usual gait test, and handgrip strength. The subjects were divided into obesity ( $\geq 30 \%$ body fat; BMI: $\left.\geq 25.0 \mathrm{~kg} / \mathrm{m}^{2}\right)$, normal-weight obesity ( $\geq 30 \%$ body fat; BMI: $\left.18.5-24.9 \mathrm{~kg} / \mathrm{m}^{2}\right)$, sarcopenia (handgrip, $<18 \mathrm{~kg}$; gait speed: $\leq 0.8 \mathrm{~m} / \mathrm{s}$; SMI: $<5.7 \mathrm{~kg} / \mathrm{m}^{2}$ ), or presarcopenia $\left(\mathrm{SMI}:<5.7 \mathrm{~kg} / \mathrm{m}^{2}\right)$. There were no subjects below the sarcopenia diagnosis criteria in usual gait speed, but not for handgrip $(0.8 \%)$ and SMI $(36.3 \%)$. Results: The prevalence of presarcopenia group (36.3\%) is higher than in the normal-weight obesity $(16.9 \%)$ and obesity $(4.8 \%)$ groups. Anthropometry and sarcopenia diagnosis assessments were significantly higher in normal-weight obesity and standard groups compared with presarcopenia group. Discussion: The number of young women was higher in the presarcopenia group than in the normal-weight obesity group, suggesting that the improvement of intrinsic skeletal muscle mass rather than fat mass is important for Japanese young women.
\end{abstract}

Keywords: normal-weight obesity, masked obesity, sarcopenia, presarcopenia, skeletal muscle index, body mass index, percent body fat, university fresh women

\section{Introduction}

For health promotion, it is important for maintaining appropriate body composition parameters (i.e., body fat, skeletal muscle, etc.) $[1,2]$. In general, excess body fat (obesity) produces an increased risk of metabolic abnormalities [3]. Recently, reports of obesity with a normal body mass index (BMI) and normal-weight obesity syndrome have increased [4]. Notably, the prevalence of normal-weight obesity syndrome has been frequently observed even in young women in recent times (approximately $18-19$ years of age) $[5,6]$. Normal-weight obesity consists of a high ratio of body fat mass and a low ratio of skeletal muscle mass and thus it is speculated that young women tend to preserve a low level of skeletal muscle mass.

Loss of skeletal muscle mass, sarcopenia, is associated with serious problems (i.e., falls, fractures, disability, and heart disease) [7-9] and it is also associated with increased healthcare costs [10]. Therefore, the European Working Group on Sarcopenia in Older People (EWGSOP) [11] and the Asian Working Group for Sarcopenia (AWGS) [12] introduced the most widely used consensus, which recommended cut-offs of skeletal muscle mass [skeletal

This is an open-access article distributed under the terms of the Creative Commons Attribution-NonCommercial 4.0 International License, which permits unrestricted use, distribution, and reproduction in any medium for non-commercial purposes, provided the original author and source are credited, a link to the CC License is provided, and changes - if any - are indicated. 
muscle index (SMI)], muscle strength (handgrip), and physical capability (usual gait speed) for diagnosing and assessing sarcopenia. Additionally, a recent study revealed that, among young adults, the prevalence of sarcopenia is higher in women than in men [13]. Thus, in the sarcopenia study, the skeletal muscle mass may be particularly important for young women. However, the relationship between the prevalence of normal-weight obesity syndrome and sarcopenia diagnosis criteria is yet unknown for young women. Thus, the purpose of this study was to examine the anthropometric, body composition, and somatotype characteristics of Japanese young women and to focus on normal-weight obesity syndrome and sarcopenia diagnosis criteria.

\section{Methods}

\section{Subjects}

A total of 125 Japanese university freshmen women (aged 18-25 years) were recruited through oral communications in a "Sports Practice" course at the School of Nursing in our university. Before informed consent was obtained, a written description of the purpose of the study and its safety was distributed to potential subjects, along with a lifestyle questionnaire. All subjects were free of overt chronic disease (e.g., diabetes, angina, myocardial infarction, cancer, and stroke) as assessed by their annual medical examination. In addition, musculoskeletal disease and knee joint surgery were also excluded in this study. As a result, 124 university freshmen women were used for data analyses. The principles of the World Medical Association Declaration of Helsinki and the American College of Sports Medicine Guidelines for Use of Human Subjects were adopted in this study. The study was approved by the Ethics Committee of Seirei Christopher University.

\section{BMI, percent body fat, and SMI}

Body mass and standing height were measured to the nearest $0.1 \mathrm{~kg}$ and $0.1 \mathrm{~cm}$, respectively, using a height scale and an electronic weight scale. BMI was defined as body mass/height ${ }^{2}\left(\mathrm{~kg} / \mathrm{m}^{2}\right)$. The multifrequency bioelectrical impedance analyzer (BIA), InBody 430 analyzer (Biospace Co., Ltd., Seoul, Korea), was used according to the manufacturer's guidelines. BIA is used to estimate the body composition according to the difference of conductivity of the various tissues based on the differences in their biological characteristics. This body composition analyzer adopts a tetrapolar, eight-point tactile electrode system that separately measures impedance of the arms, trunk, and legs at three different frequencies $(5,50$, and $250 \mathrm{kHz})$ for each segment. The measurements were carried out while the subjects rested quietly in the supine position, with their elbows extended and relaxed along their trunk. The InBody automatically estimates weight, BMI, percent body fat, and lean soft tissue of the two upper limbs and two lower limbs. SMI [appendicular muscle mass (AMM)/height $\left.{ }^{2}\left(\mathrm{~kg} / \mathrm{m}^{2}\right)\right]$ was calculated as the sum of the two upper limbs and two lower limbs (AMM). We divided BMI into two categories $\left(<25 \mathrm{~kg} / \mathrm{m}^{2}\right.$ and $\left.\geq 25 \mathrm{~kg} / \mathrm{m}^{2}\right)$, percent body fat into two categories $(<30 \%$ and $\geq 30 \%)$, and defined a low SMI as a value $<5.7 \mathrm{~kg} / \mathrm{m}^{2}$ in women according to the normal-weight obesity $[3,5,6]$ and the AWGS criteria [12].

\section{Usual gait test}

The usual gait test was measured over a 6-m course [11].

\section{Handgrip strength}

Handgrip strength was measured using a factorycalibrated hand dynamometer (TKK 5401, Takei, Tokyo, Japan). All subjects were instructed to maintain an upright standing position, arms at their side, and holding the dynamometer in the right hand with the elbow extended at $180^{\circ}$ without squeezing their arm against their body. The size of the dynamometer's handle was set to that which felt comfortable for the subject while squeezing the grip. Each subject underwent two trials, and the best value of the trials was used for analysis.

\section{Definition of obesity, normal-weight obesity, sarcopenia, and presarcopenia}

In this study, "obesity" ( $\geq 30 \%$ body fat; BMI: $\geq 25.0 \mathrm{~kg} / \mathrm{m}^{2}$ ) and "normal-weight obesity" groups $(\geq 30 \%$ body fat; BMI: $18.5-24.9 \mathrm{~kg} / \mathrm{m}^{2}$ ) were defined according to the previous study $[3,5,6]$. "Sarcopenia" (handgrip, $<18 \mathrm{~kg}$; gait speed: $\left.\leq 0.8 \mathrm{~m} / \mathrm{s} ; \mathrm{SMI}:<5.7 \mathrm{~kg} / \mathrm{m}^{2}\right)$ and "presarcopenia" (SMI: $\left.<5.7 \mathrm{~kg} / \mathrm{m}^{2}\right)$ groups were defined according to the EWGSOP and the AWGS criteria $[11,12]$.

\section{Statistical analyses}

The results are expressed as mean \pm standard deviation for all variables. All data were analyzed using JMP software v.12.0 for Mac (SAS Institute Inc., Tokyo, Japan). We excluded the individuals $(n=8)$ with obesity group $(\geq 30 \%$ body fat, $n=6)$ and combined the normal-weight obesity and presarcopenia groups $\left(\geq 30 \%\right.$ body fat and $<5.7 \mathrm{~kg} / \mathrm{m}^{2}$, $n=2)$, because these groups were extremely small samples compared to the normal-weight obesity ( $\geq 30 \%$ body fat, $n=19)$, presarcopenia $\left(<5.7 \mathrm{~kg} / \mathrm{m}^{2}, n=43\right)$, and other 
(standard, $n=54$ ) groups. When the data were not normally distributed, non-parametric statistical analysis (Wilcoxon signed-rank test) was used to identify differences in normal-weight obesity, presarcopenia, and standard groups. Statistical significance was set at $p<0.05$.

\section{Results}

Handgrip, SMI, and usual gait were $26.4 \pm 4.0$ (ranging from 15.7 to 36.5 ) $\mathrm{kg}, 5.93 \pm 0.56$ (ranging from 4.61 to 7.85 ) $\mathrm{kg} / \mathrm{m}^{2}$, and $1.43 \pm 0.41$ (ranging from 0.88 to $4.55) \mathrm{m} / \mathrm{s}$, respectively. There were no subjects below the sarcopenia diagnosis criteria regarding usual gait speed, but that was not true for handgrip $(0.8 \%, n=1)$ and SMI $(36.3 \%, n=45)$. The relationship between BMI and percent body fat $(r=0.845, p<0.01)$ for 124 university freshmen women is shown in Fig. 1 . The prevalence of presarcopenia group $(36.3 \%)$ was higher than in the normal-weight obesity $(16.9 \%)$ and obesity $(4.8 \%)$ groups. The prevalence of combining both normalweight obesity and presarcopenia was $1.6 \%$ (Fig. 2).

Anthropometry and sarcopenia diagnosis assessments were significantly higher in the normal-weight obesity and standard groups compared with the presarcopenia group (Table I). Anthropometry assessments were significantly higher in the normal-weight obesity group than in the standard group, whereas there were no differences in sarcopenia diagnosis assessments between the two groups.

\section{Discussion}

Although throughout the world there is a lack of consensus in the percent body fat regarding normal-weight obesity diagnostic criteria, this study used the level of percent body

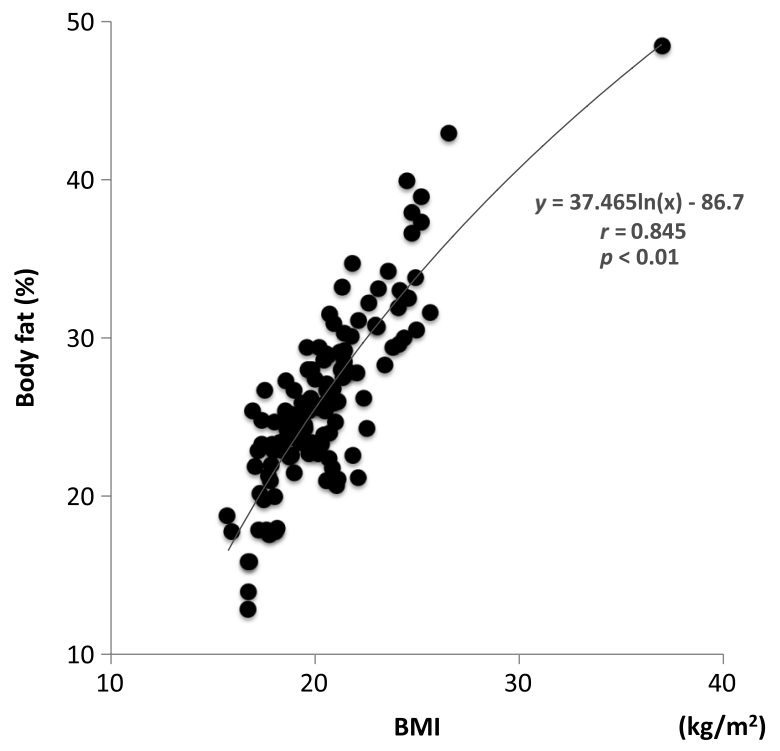

Fig. 1. Relationship between body mass index (BMI) and percent body fat in healthy Japanese young women

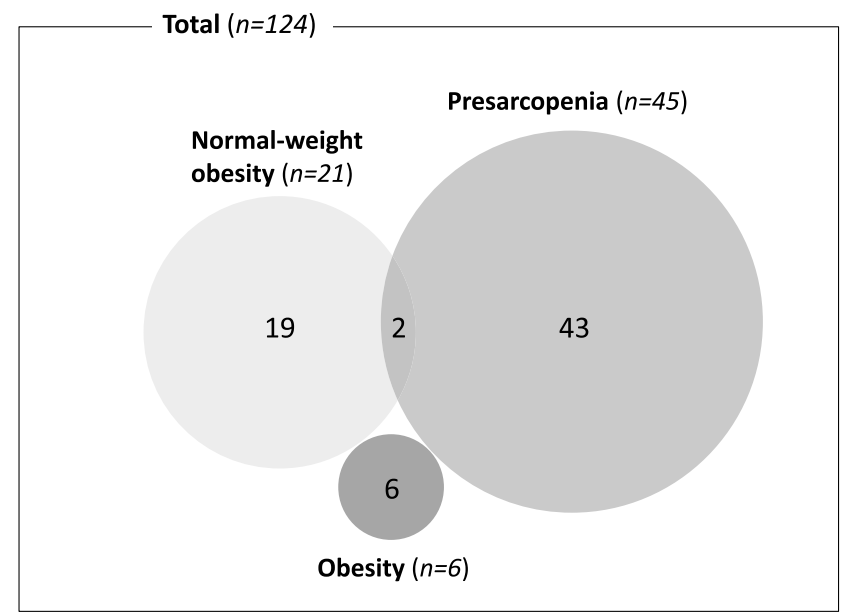

Fig. 2. The number of obesity, normal-weight obesity, and presarcopenia in healthy Japanese young women $(n=124)$

fat and BMI ( $\geq 30 \%$ body fat; BMI: $18.5-24.9 \mathrm{~kg} / \mathrm{m}^{2}$ ) in women based on previous Japanese studies as a reference $[5,6]$. The prevalence of normal-weight obesity in this study $(16.9 \%)$ was almost exactly the same as in the previous studies $(12.3 \%$ and $17.5 \%$, respectively) $[5,6]$. In addition, the handgrip was similar between this study (26.4 $\mathrm{kg}$ for 18.4 years) and previous national survey (approximately $27 \mathrm{~kg}$ for 19 years) [14]. Thus, it appears that the body composition and somatotype characteristics as well as muscle function in this study demonstrate the present situation as a general population of healthy Japanese young women.

In this study, skeletal muscle morphology and function were greater in normal-weight obesity and standard groups compared with the presarcopenia group. In addition, the prevalence of combined normal-weight obesity and presarcopenia group was very rare (1.6\%). This means that healthy Japanese young women with normal-weight obesity syndrome are not entirely correctly labeled as "presarcopenia." Recently, a previous study reported that skeletal muscle loss is not associated with a metabolic phenotype in men and women with obesity [15]. Taken together, there is a high possibility that a countermeasure against presarcopenia as well as normal-weight obesity is required.

Interestingly, this study demonstrated that the number of young women was approximately twofold greater in the presarcopenia group $(36.3 \%)$ compared to the normalweight obesity group (16.9\%). Since the low skeletal muscle mass may affect the warning signs of suppressed skeletal muscle thermogenesis, insulin resistance, diabetes, and metabolic syndrome $[16,17]$, this indicates that considerable attention should be made even in healthy young women. In addition, muscle strength and physical capability were not below the sarcopenia diagnosis criteria in the presarcopenia group, but handgrip and usual gait speed as well as skeletal muscle mass were significantly lower in the presarcopenia group compared with 
Table I Anthropometry and sarcopenia diagnosis assessments in female university freshmen $(n=124)$

\begin{tabular}{|c|c|c|c|}
\hline Variable & Normal-weight obesity & Presarcopenia & Standard \\
\hline$N$ & 19 & 43 & 54 \\
\hline Age (years) & $18.4(1.4)$ & $18.2(0.4)$ & $18.6(1.1)$ \\
\hline \multicolumn{4}{|l|}{ Anthropometry assessments } \\
\hline Height (m) & $158.0(5.9)^{*}$ & $154.7(3.2)$ & $159.3(5.3)^{* *}$ \\
\hline Weight $(\mathrm{kg})$ & $58.5(5.5)^{* *}, \#$ & $43.8(3.2)$ & $52.0(4.3)^{* *}$ \\
\hline Body mass index $\left(\mathrm{kg} / \mathrm{m}^{2}\right)$ & $23.4(1.2)^{* *}, \#$ & $18.3(1.2)$ & $20.5(1.5)^{* *}$ \\
\hline Body fat $(\%)$ & $33.1(2.7)^{* *}, \#$ & $23.3(3.8)$ & $24.6(3.4)$ \\
\hline Upper-leg $50 \%$ girth $(\mathrm{cm})$ & $53.5(2.5)^{* *}, \#$ & $45.1(1.9)$ & $49.0(2.6)^{* *}$ \\
\hline Lower-leg $30 \%$ girth $(\mathrm{cm})$ & $36.6(1.8)^{* *}, \#$ & $32.1(1.3)$ & $34.6(1.6)^{* *}$ \\
\hline \multicolumn{4}{|c|}{ Sarcopenia diagnosis assessments } \\
\hline Handgrip (kg) & $26.7(3.0)^{* *}$ & $24.4(2.5)$ & $28.0(4.3)^{* *}$ \\
\hline Skeletal MM $\left(\mathrm{kg} / \mathrm{m}^{2}\right)$ & $6.30(0.39)^{* *}$ & $5.37(0.24)$ & $6.16(0.38)^{* *}$ \\
\hline Usual gait speed (m/s) & $1.52(0.48)^{*}$ & $1.32(0.21)$ & $1.49(0.50)^{*}$ \\
\hline
\end{tabular}

MM: muscle mass.

${ }^{\star} p<0.05$ vs. presarcopenia.

${ }^{\star *} p<0.01$ vs. presarcopenia.

${ }^{\#} p<0.01$ vs. standard

normal-weight obesity and standard groups. These results suggested that the improvement of intrinsic skeletal muscle mass rather than fat mass is important for healthy Japanese young women.

The limitation of this study should be discussed. As the subjects were Japanese university freshmen women, the age distribution and the physical characteristics were very limited. Additional research studying these issues is needed.

In conclusion, the prevalence was higher in the presarcopenia group than in the normal-weight obesity group for Japanese university freshmen women, suggesting that the improvement of intrinsic skeletal muscle mass rather than fat mass is important for Japanese young women.

Funding sources: This study was partially supported by Grant-in-aid (nos. 15K01553 and 18K10906) from the Japan Ministry of Education, Culture, Sports, Science, and Technology and Seirei Christopher University Grants-in-Aid of Research.

Author's contribution: TY contributed to study design, acquisition of data, drafting of the manuscript, literature review, and critical revision of the manuscript. The author takes responsibility for the integrity and the accuracy of the data.

Conflict of interest: The author declares that he has no financial or personal conflict of interest with regard to this study.

Acknowledgements: The author would like to thank the individuals who participated in this study.

\section{References}

1. Gill LE, Bartels SJ, Batsis JA: Weight management in older adults. Curr Obes Rep 4, 379-388 (2015)
2. Bae S, Shimada H, Park H, Lee S, Makizako H, Doi T, Yoshida D, Tsutsumimoto K, Anan Y, Suzuki T: Association between body composition parameters and risk of mild cognitive impairment in older Japanese adults. Geriatr Gerontol Int 17, 2053-2059 (2017)

3. Kim JY, Han SH, Yang BM: Implication of high-body-fat percentage on cardiometabolic risk in middle-aged, healthy, normal-weight adults. Obesity 21, 1571-1577 (2013)

4. Franco LP, Morais CC, Cominetti C: Normal-weight obesity syndrome: Diagnosis, prevalence, and clinical implications. Nutr Rev 74, 558-570 (2016)

5. Inoue $\mathrm{H}, \mathrm{Kuwano} \mathrm{T}$ : Effects of blood parameters and dietary intake on Japanese female college students showing normal BMI with high body fat. J Home Economics Jpn 63, 421-430 (2012)

6. Yamaguchi-Tanaka Y, Kawagoshi Y, Sasaki S, Fukao A: Crosssectional study of possible association between rapid eating and high body fat rates among female Japanese college students. J Nutr Sci Vitaminol 59, 243-249 (2013)

7. Visser M, Goddpaster BH, Kritchevsky SB, Newman AB, Nevitt M, Rubin SM, Simonsick EM, Harris TB: Muscle mass, muscle strength, and muscle fat infiltration as predictors of incident mobility limitations in well-functioning older persons. J Gerontol A Biol Sci Med Sci 60, 324-333 (2005)

8. Haykowsky MJ, Brubaker PH, Morgan TM, Kritchevsky S, Eggebeen J, Kitzman DW: Impaired aerobic capacity and physical functional performance in older heart failure patients with preserved ejection fraction: Role of lean body mass. J Gerontol A Biol Sci Med Sci 68, 968-975 (2013)

9. Yasuda T, Nakajima T, Sawaguchi T, Nozawa N, Arakawa T, Takahashi R, Mizushima Y, Katayanagi S, Matsumoto K, Toyoda $S$, Inoue T: Short physical performance battery for cardiovascular disease inpatients: Implications for critical factors and sarcopenia. Sci Rep 7, 17425 (2017)

10. Janssen I, Shepard DS, Katzmarzyk PT, Roubenoff R: The healthcare costs of sarcopenia in the United States. J Am Geriatr Soc 52, 80-85 (2004)

11. Cruz-Jentoft AJ, Baeyens JP, Bauer JM, Boirie Y, Cederholm T, Landi F, Martin FC, Michel JP, Rolland Y, Schneider SM, Topinková E, Vandewoude M, Zamboni M, European Working 
Group on Sarcopenia in Older People: Sarcopenia: European consensus on definition and diagnosis: Report of the European working group on Sarcopenia in older people. Age Ageing 39, 412$423(2010)$

12. Chen LK, Liu LK, Woo J, Assantachai P, Auyeung TW, Bahyah KS, Chou MY, Chen LY, Hsu PS, Krairit O, Lee JS, Lee WJ, Lee Y, Liang CK, Limpawattana P, Lin CS, Peng LN, Satake S, Suzuki T, Won CW, Wu CH, Wu SN, Zhang T, Zeng P, Akishita M, Arai H: Sarcopenia in Asia: Consensus report of the Asian Working Group for Sarcopenia. J Am Med Dir Assoc 15, 95-101 (2014)

13. Yamada M, Nishiguchi S, Fukutani N, Tanigawa T, Yukutake T, Kayama H, Aoyama T, Arai H: Prevalence of sarcopenia in community-dwelling Japanese older adults. J Am Med Dir Assoc 14, 911-915 (2013)
14. Ministry of Education, Culture, Sports, Science and Technology (MEXT): Results of the FY2013 Physical Fitness Survey (Japan Fitness Test). Retrieved from http://www.mext.go.jp/en/ news/topics/detail/1372622.htm. Accessed on: March 24, 2019

15. Hwang YC, Cho IJ, Jeong IK, Ahn KJ, Chung HY: Differential association between sarcopenia and metabolic phenotype in Korean young and older adults with and without obesity. Obesity (Silver Spring) 25, 244-251 (2017)

16. Dulloo AG: A role for suppressed skeletal muscle thermogenesis in pathways from weight fluctuations to the insulin resistance syndrome. Acta Physiol Scand 184, 295-307 (2005)

17. Moon SS: Low skeletal muscle mass is associated with insulin resistance, diabetes, and metabolic syndrome in the Korean population: The Korea National Health and Nutrition Examination Survey (KNHANES) 2009-2010. Endocr J 61, 61-70 (2014) 